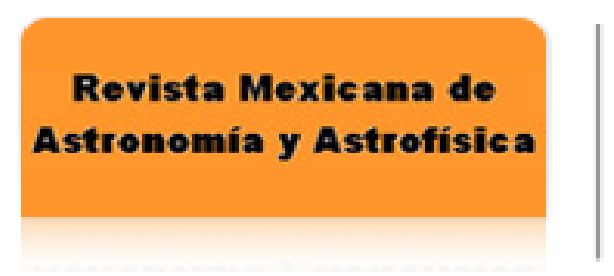

Revista Mexicana de Astronomía y Astrofísica ISSN: 0185-1101

rmaa@astroscu.unam.mx

Instituto de Astronomía

México

Lora, V.; Vasconcelos, M. J.; Raga, A. C.; Esquivel, A.; Cerqueira, A. H.

THE PHOTOEVAPORATION OF A NEUTRAL STRUCTURE BY AN EUV+FUV RADIATION FIELD

Revista Mexicana de Astronomía y Astrofísica, vol. 49, núm. 2, octubre, 2013, pp. 275-284

Instituto de Astronomía

Distrito Federal, México

Available in: http://www.redalyc.org/articulo.oa?id=57128966009

How to cite

- Complete issue

- More information about this article

Journal's homepage in redalyc.org

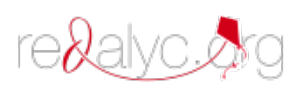

Scientific Information System Network of Scientific Journals from Latin America, the Caribbean, Spain and Portugal Non-profit academic project, developed under the open access initiative 


\title{
THE PHOTOEVAPORATION OF A NEUTRAL STRUCTURE BY AN EUV+FUV RADIATION FIELD
}

\author{
V. Lora,${ }^{1}$ M. J. Vasconcelos, ${ }^{2}$ A. C. Raga,${ }^{3}$ A. Esquivel,${ }^{3}$ and A. H. Cerqueira ${ }^{2}$ \\ Received 2013 February 22; accepted 2013 June 12
}

\begin{abstract}
RESUMEN
La radiación fotoionizante EUV y la radiación fotodisociante FUV de estrellas nacientes fotoevaporan la nube donde nacen, llevando a la formación de grumos densos que eventualmente podrían formar estrellas adicionales. Estudiamos los efectos de incluir un flujo fotodisociante FUV, en modelos de fragmentación de una nube molecular fotoevaporada y autogravitante. Llevamos a cabo simulaciones 3D de la interacción de una nube inhomogénea, neutra y autogravitante, con una radiación externa de campos EUV y FUV, y calculamos el número y la masa de los grumos que están en colapso. Encontramos que la presencia de una región de fotodisociación entre la región HII y la nube molecular tiene un efecto importante en la formación de estructuras densas debido a la expansión de una región HII. En particular, incluir un campo FUV lleva a una formación más temprana de un número mayor de grumos densos, los cuales podrían llevar a la formación de un número mayor de estrellas.
\end{abstract}

\begin{abstract}
The EUV photoionizing radiation and FUV dissociating radiation from newly born stars photoevaporate their parental neutral cloud, leading to the formation of dense clumps that could eventually form additional stars. We study the effects of including a photodissociating FUV flux in models of the fragmentation of a photoevaporating, self-gravitating molecular cloud. We compute 3D simulations of the interaction of an inhomogeneous, neutral, self-gravitating cloud with external EUV and FUV radiation fields, and calculate the number of collapsing clumps and their mass. We find that the presence of an outer photodissociation region has an important effect on the formation of dense structures due to the expansion of an H II region. In particular, including a FUV field leads to the earlier formation of a larger number of dense clumps, which might lead to the formation of more stars.

Key Words: HII regions - ISM: clouds - ISM: kinematics and dynamics — stars: formation
\end{abstract}

\section{INTRODUCTION}

The expansion of an HII region into a surrounding, inhomogeneous molecular cloud leads to the formation of complex, elongated "elephant trunk" structures. Examples of this are the trunks in the Eagle (Hester et al. 1996) and the Rosette (Carlqvist, Gahm, \& Kristen 2003) nebulae. Examples of similar (but larger) structures in external galaxies have been found as well (Carlqvist 2010).

\footnotetext{
${ }^{1}$ Astronomisches Rechen-Institut, Heidelberg, Germany.

${ }^{2}$ Laboratório de Astrofísica Teórica e Observacional, Bahia, Brazil.

${ }^{3}$ Instituto de Ciencias Nucleares, Universidad Autónoma de México, Mexico.
}

A considerable amount of theoretical work has focused on the photoevaporation of a single, dense clump, starting with the paper of Oort \& Spitzer (1955). This problem has been addressed both analytically (Bertoldi 1989; Bertoldi \& McKee 1990) and numerically (Lefloch \& Lazareff 1994; Dale, Bonnell, \& Whitworth 2007; Dale, Clark, \& Bonnell 2007; Dale \& Bonell 2011; Ercolano et al. 2012). Several simulations have included detailed treatments of the radiative transfer and ionization (Mellema et al. 1998; Raga et al. 2009), and the study of clumps with low amplitude density inhomogeneities (González, Raga, \& Steffen 2005), with self-gravity (Esquivel \& Raga 2007) and with magnetic fields (Henney et 
al. 2009). These models are applicable to photoionized regions in which individual neutral clumps are clearly visible (the evident example of this being the Helix Nebula, see, e.g., O'Dell, Henney, \& Ferland 2005).

However, the observations of elephant trunks suggest the presence of more complex density structures in the region with neutral gas. Attempts to address this have included 3D simulations of the propagation of ionization fronts into "multi-clump" structures (Lim \& Mellema 2003; Raga et al. 2009; Mackey \& Lim 2010, 2011) and into more complex density distributions (Mellema et al. 2006; Esquivel \& Raga 2007; Mac Low et al. 2007; Gritschneder et al. 2009; Ercolano \& Gritschneder 2011; Arthur et al. 2011). These simulations took into account the following physical processes:

- direct gas dynamics+ionizing radiation transfer (Lim \& Mellema 2003; Mackey \& Lim 2010),

- self-gravity (Esquivel \& Raga 2007; Mac Low et al. 2007; Gritschneder et al. 2010; Ercolano \& Gritschneder 2011),

- magnetic fields (Mackey \& Lim 2011; Arthur et al. 2011),

- the diffuse ionizing radiation field (Raga et al. 2009; Ercolano \& Gritschneder 2011).

An important effect that has not been explored in detail until very recently is the presence of a photodissociating FUV radiation field (together with the photoionizing EUV radiation). Arthur et al. (2011) presented numerical simulations including the FUV radiation field and obtained that the FUV field can have a clear dynamical importance in the formation of dense clumps at the edge of an expanding HII region. Their simulations include the presence of a magnetic field, but do not include the self-gravity of the gas.

In the present paper, we discuss 3D simulations which include the transfer of the EUV and FUV radiation in a self-gravitating medium with an inhomogeneous initial density distribution. Our simulations differ from those of Arthur et al. (2011) in that they do not include a magnetic field, but do consider the self-gravity of the gas (see $\S 2$ ).

We then use the results of our simulations to calculate the number of clumps $(\S 3)$ and mass distributions of the dense clumps $(\S 4)$. Finally, the results are summarized in $\S 5$.

\section{THE NUMERICAL SIMULATIONS}

We have carried out twenty-four 3D simulations with the code described by Lora, Raga, \& Esquivel (2009). This code integrates the gasdynamic equations in a uniform 3D, Cartesian grid, together with the radiative transfer of radiation at the Lyman limit, and a hydrogen ionization rate equation, including the self-gravity of the gas.

The radiative transfer and hydrogen ionization are solved as follows. We place an ionizing photon source (producing $S_{*}$ ionizing photons per unit time) far away, outside the computational grid, and then impose an ionizing photon flux $F_{0}=S_{*} /\left(4 \pi R_{0}^{2}\right)$ on the boundary of the computational grid, where $R_{0}$ is the distance from the grid boundary to the photon source (assumed to lie along the $x$-axis). The ionizing photon flux is then marched into the computational domain as:

$$
F_{i+1, j, k}=F_{i, j, k} e^{-\Delta \tau_{\mathrm{Ly}}},
$$

where $F_{i, j, k}$ is the ionizing photon flux at the left boundary (along the $x$-axis) of the $(i, j, k)$ computational cell and

$$
\Delta \tau_{\mathrm{Ly}}=n_{\mathrm{HI}}\left(\sigma_{\mathrm{H}, \nu_{0}}+\sigma_{d}\right) \Delta x
$$

where $n_{\mathrm{HI}}$ is the neutral $\mathrm{H}$ density of cell $(i, j, k)$, $\Delta x$ is the size of the cell (along the $x$-axis), $\sigma_{\mathrm{H}, \nu_{0}}=$ $6.30 \times 10^{-18} \mathrm{~cm}^{2}$ is the Lyman limit photoionization cross section of $\mathrm{HI}$ and $\sigma_{d}=1.1 \mathrm{~cm}^{2}$ is the FUV/EUV dust absorption cross section. This value of $\sigma_{d}$ is derived assuming that the EUV/FUV dust absorption is $A=1.2 \times 10^{-21} \mathrm{~cm}^{-2} N_{\mathrm{HI}}$ (the derivation of this relation is discussed by Vasconcelos, Cerqueira, \& Raga 2011). We should note that in calculating the Lyman limit optical depth through equation (2) we are assuming that the region of photoionized $\mathrm{H}$ has no dust. If it did, one would have to replace $n_{\mathrm{HI}}$ by $n_{\mathrm{H}}$ (the total $\mathrm{H}$ number density) in equation (2). However, because of the low column densities of the ionized $\mathrm{H}$ regions within our computational domain, it makes no difference whether or not dust is present in these regions.

With the ionizing photon flux $F$, we calculate the $\mathrm{H}$ photoionization rate

$$
\phi_{\mathrm{H}}=\left(1-e^{-\Delta \tau_{\mathrm{Ly}}}\right) F .
$$

This photoionization rate is then included in a $\mathrm{HI}$ continuity equation:

$$
\frac{\partial n_{\mathrm{HI}}}{\partial t}+\dot{\nabla}\left(n_{\mathrm{HI}} \underline{u}\right)=n_{e} n_{\mathrm{HII}} \alpha_{\mathrm{H}}(T)-n_{\mathrm{HI}} \phi_{\mathrm{H}},
$$

where $n_{\mathrm{HI}}, n_{\mathrm{HII}}$ and $n_{e}$ are the neutral $\mathrm{H}$, ionized $\mathrm{H}$ and electron densities (respectivey) and $\alpha_{\mathrm{H}}(T)$ is the 
recombination coefficient of $\mathrm{H}$. This equation is integrated together with the standard 3D gasdynamic equations. The diffuse radiation is included only by considering the "case B" recombination (to all levels with energy quantum number $N>1$ ).

To equations (1-4), which were included in the code of Lora et al. (2009), we have now added the transfer of FUV radiation, and an ionization rate equation for CI. This has been done in the following way. For the FUV photons, we solve the radiative transfer problem at $\lambda=1100 \AA$ (the ionization edge of the ground state of $\mathrm{CI}$ ), considering the absorption due to CI photoionization and to dust extinction. We therefore calculate the optical depth for the FUV photons in each computational cell as:

$$
\Delta \tau_{\mathrm{FUV}}=\left(n_{\mathrm{CI}} \sigma_{\mathrm{C}, \nu_{0}}+n_{\mathrm{HI}} \sigma_{d}\right) \Delta x,
$$

where $n_{\mathrm{CI}}$ is the CI number density and $\sigma_{\mathrm{C}, \nu_{0}}=$ $1.22 \times 10^{-17} \mathrm{~cm}^{2}$ is the photoionization cross section at the CI ionization edge. We have assumed that the dust extinction cross section (per $\mathrm{H}$ atom) has the same values at the $\mathrm{CI}$ and HI ionization edges (i.e. and $\lambda \sim 1100$ and $900 \AA$, respectively). With this value for the FUV optical depth of the computational cells, we then use equations equivalent to equation (1) and equation (3) to calculate the CI photoionization rate. We then integrate a continuity equation for CI (with the appropriate source terms, such as equation 4 but for CI) together with the gasdynamic and HI continuity equations.

Instead of integrating an energy equation (with appropriate heating and cooling terms), we compute the temperature of the gas as:

$$
T=\left(T_{1}-T_{2}\right) x_{\mathrm{HII}}+T_{2} x_{\mathrm{CII}}+T_{3}\left(1-x_{\mathrm{CII}}\right),
$$

where $x_{\mathrm{HII}}$ and $x_{\mathrm{CII}}$ are the $\mathrm{H}$ and $\mathrm{C}$ ionization fractions (respectively), and $T_{1}=10^{4} \mathrm{~K}, T_{2}=10^{3} \mathrm{~K}$ and $T_{3}=10 \mathrm{~K}$ are the typical temperatures of photoionized, photodissociated and molecular regions respectively. Therefore, we do not calculate the photodissociation of $\mathrm{H}_{2}$, and assume following Richling \& Yorke (2000), that it approximately follows the ionization of CI.

From the paper of Díaz-Miller, Franco, \& Shore (1998), we now take the EUV $(\lambda>912 \AA)$ photon rate $S_{\mathrm{I}}$ and the FUV $(912 \AA<\lambda<1100 \AA)$ photon rate $S_{\mathrm{D}}$ for a set of three main sequence $\mathrm{O}$ stars (of effective temperatures $T=50000,45000$ and $40000 \mathrm{~K}$ ). For the EUV photons, we solve the radiative transfer problem (parallel to the $x$-axis of the computational grid) using the Lyman-limit $\mathrm{H}$ absorption coefficient $\sigma_{\nu_{0}}(\mathrm{H})=6.30 \times 10^{-18} \mathrm{~cm}^{2}$, and using the EUV flux to calculate the HI photoionization rate (this is completely equivalent to the models of Lora et al. 2009).

For our simulations, we consider a computational domain of $(3.0,1.5,1.5) \times 10^{18} \mathrm{~cm}$ (along the $x$-, $y$ - and $z$-axes, respectively) resolved with $256 \times 128 \times 128$ grid points. An outflow boundary condition is applied on the $x$-axis boundaries, and reflection conditions in all of the other boundaries.

This domain is initially filled with an inhomogeneous density structure with a power law powerspectrum index of $-11 / 3$ (i.e., $P(k) \propto k^{-11 / 3}$, where $k$ is the wave number, see Esquivel et al. 2003). This results in a density distribution with a dispersion of $\approx 2$ times the mean density. We have chosen four different realizations of the density distribution, which we use to compute models identified with the letters $\mathrm{M}, \mathrm{O}, \mathrm{D}$ and $\mathrm{E}$ (each letter corresponding to one of the chosen initial density distributions).

The medium is initially at rest. The computational domain is divided into an initially ionized region (with ionized $\mathrm{H}$ and $\mathrm{C}$ ) for $x<x_{0}=4 \times 10^{17} \mathrm{~cm}$ and a neutral region (with neutral $\mathrm{H}$ and $\mathrm{C}$ ) for $x>$ $x_{0}$. The average density in the neutral medium is 100 times the average density in the ionized medium, and the transition between the two follows a tanh profile with a width of $\sim 10$ pixels. The resulting neutral structure has a total mass of $228 M_{\odot}$. This initial setup is identical to the one used by Lora et al. (2009).

Other authors have used more complex initial conditions for this kind of simulation, in particular, including an initial velocity field. This was done, e.g., by Arthur et al. (2011), who took as initial conditions the output from a 3D, turbulent cloud simulation. Other examples of initial conditions can be found in Dale \& Bonell (2011), Dale \& Bonell (2012) and Ercolano et al. (2012). However, as the flow motions induced by the photoionization and photoevaporation have velocities which are much larger than the ones of the initial turbulent motions, including these initial motions is unlikely to produce large effects on the results.

We assume that we have a stellar source situated $3 \times 10^{18} \mathrm{~cm}$ from the edge of the computational domain in the $-x$ direction. For this photon source we consider three possibilities, an O3 $\left(T_{\text {eff }}=50000 \mathrm{~K}\right)$, an O5.5 $\left(T_{\text {eff }}=45000 \mathrm{~K}\right)$ and an O7.5 $\left(T_{\text {eff }}=40000 \mathrm{~K}\right)$ main sequence star. The EUV and FUV fluxes computed by Díaz-Miller et al. (1998) for such stars are given in Table 1.

We then compute simulations with the EUV and FUV fluxes given in Table 1 (models M1-3, O1-3, 
TABLE 1

MODEL PARAMETERS

\begin{tabular}{|c|c|c|c|c|}
\hline Models & $\begin{array}{c}T_{\text {eff }} \\
10^{3} \mathrm{~K}\end{array}$ & $\begin{array}{l}\log _{10} S_{\mathrm{I}} \\
\text { photo }\end{array}$ & $\begin{array}{l}\log _{10} S_{\mathrm{D}} \\
\mathrm{ns} \mathrm{s}^{-1}\end{array}$ & $S_{\mathrm{D}} / S_{\mathrm{I}}$ \\
\hline $\mathrm{M} 1, \mathrm{O} 1, \mathrm{D} 1, \mathrm{E} 1^{\mathrm{a}, \mathrm{b}}$ & 50 & 49.89 & 49.54 & 0.45 \\
\hline $\mathrm{M} 2, \mathrm{O} 2, \mathrm{D} 2, \mathrm{E} 2^{\mathrm{a}, \mathrm{b}}$ & 45 & 49.35 & 49.16 & 0.65 \\
\hline $\mathrm{M} 3, \mathrm{O} 3, \mathrm{D} 3, \mathrm{E} 3^{\mathrm{a}, \mathrm{b}}$ & 40 & 48.78 & 48.76 & 0.96 \\
\hline
\end{tabular}

${ }^{a}$ Models with letters $M$ through E have identical FUV and EUV fluxes, and correspond to different initial density distributions (see the text).

${ }^{\mathrm{b}}$ Models M1B-E1B, M2B-E2B and M3B-E3B have the same values of $S_{I}$ as models M1-E1, M2-E2 and M3-E3 (respectively), but have $S_{D}=0$.

D1-3 and E1-3, with letter M-E corresponding to the four different initial density distributions, see above), and simulations with the same EUV fluxes but with zero FUV flux (models M1B-3B, O1B-3B, D1B-3B and E1B-3B). As can be seen from Table 1, the models with the same number (second character of the model identification) have the same impinging EUV field. The set of models with zero FUV fields (models M1B-E3B) has a transition from $T_{3}=10 \mathrm{~K}$ in the region with neutral $\mathrm{H}$ to $T_{1}=10^{4} \mathrm{~K}$ in the photoionized region (see equation 6 ), with no intervening photodissociated region, and are therefore equivalent to the models of Lora et al. (2009).

\section{RESULTS}

In Figures 1 and 2, we show the mid-plane density stratifications and the positions of the $\mathrm{H}$ and $\mathrm{C}$ ionization fronts obtained for a $t=2.5 \mathrm{kyr}$ (Figure 1 ) and $t=100 \mathrm{kyr}$ (Figure 2) integration time for models M1, O1, D1 and E1. In Figures 3 and 4, we show the same density stratifications, obtained for a $t=2.5 \mathrm{kyr}$ (Figure 3 ) and $t=100 \mathrm{kyr}$ (Figure 4 ) integration time for the models with zero FUV fluxes: M1B, O1B, D1B and E1B.

In the models with non-zero FUV fluxes (see Figures 1 and 2) we see that the $\mathrm{C}$ and $\mathrm{H}$ ionization fronts are separated by a photodissociated region (with CII and HI) with a width of $\sim 10^{18} \mathrm{~cm}$ in the $t=2.5$ and 100 kyr frames. The neutral region (to the right of the $\mathrm{C}$ ionization front) develops progressively denser regions which collapse under the combined effects of the compression ahead of the $\mathrm{C}$ ionization front and the self-gravity of the gas. As expected, the higher temperature, photodissociated region does not develop such dense structures.

For the models with zero FUV fluxes (models M1B-E1B, see Figures 3 and 4) there is of course no photodissociated region.
Qualitatively similar time-evolutions are obtained for all of the computed models. Of course, the details of the fragmentation of the neutral gas into dense clumps differ in all simulations. These differences are quantified in the following subsection.

\subsection{Number of clumps as a function of time}

We take the density stratifications resulting from our 24 simulations (see Table 1), and compute the number of clumps present at different integration times. To calculate the number of clumps, we define a cutoff density $\rho_{c}$, and count all spatially contiguous structures with densities $\rho \geq \rho_{c}$.

Following Lora et al. (2009), we choose three different cutoff densities $\rho_{c}=10^{-20}, 10^{-19}$ and $3 \times$ $10^{-18} \mathrm{~g} \mathrm{~cm}^{-3}$ (corresponding to number densities of atomic nuclei $\sim 4600,46000$ and $\left.1.4 \times 10^{6} \mathrm{~cm}^{-3}\right)$.

For the models without a FUV field (models M1B-E3B), we count clumps in the neutral $\mathrm{H}$ region. For the models with non-zero FUV fields (models M1-E3, see Table 1), we count clumps that satisfy one of the two following conditions:

1. that their material has neutral $\mathrm{H}$,

2. that they have neutral C.

Notably, these two criteria result in identical clump numbers for the $\rho_{c}=10^{-19}$ and $3 \times 10^{-18} \mathrm{~g} \mathrm{~cm}^{-3}$ cutoff densities (see Figures 5, 6, and 7). For $\rho_{c}=$ $10^{-20} \mathrm{~g} \mathrm{~cm}^{-3}$, the second criterion results in somewhat larger clump numbers. In the computation of the clump numbers, we calculate averages over each of the sets of four models with identical parameters (see Table 1) but with different initial density structures.

The results obtained from this clump counting exercise are given in Figure 5 (models M1-E1 and M1B-E1B), Figure 6 (models M2-E2 and M2B-E2B) 


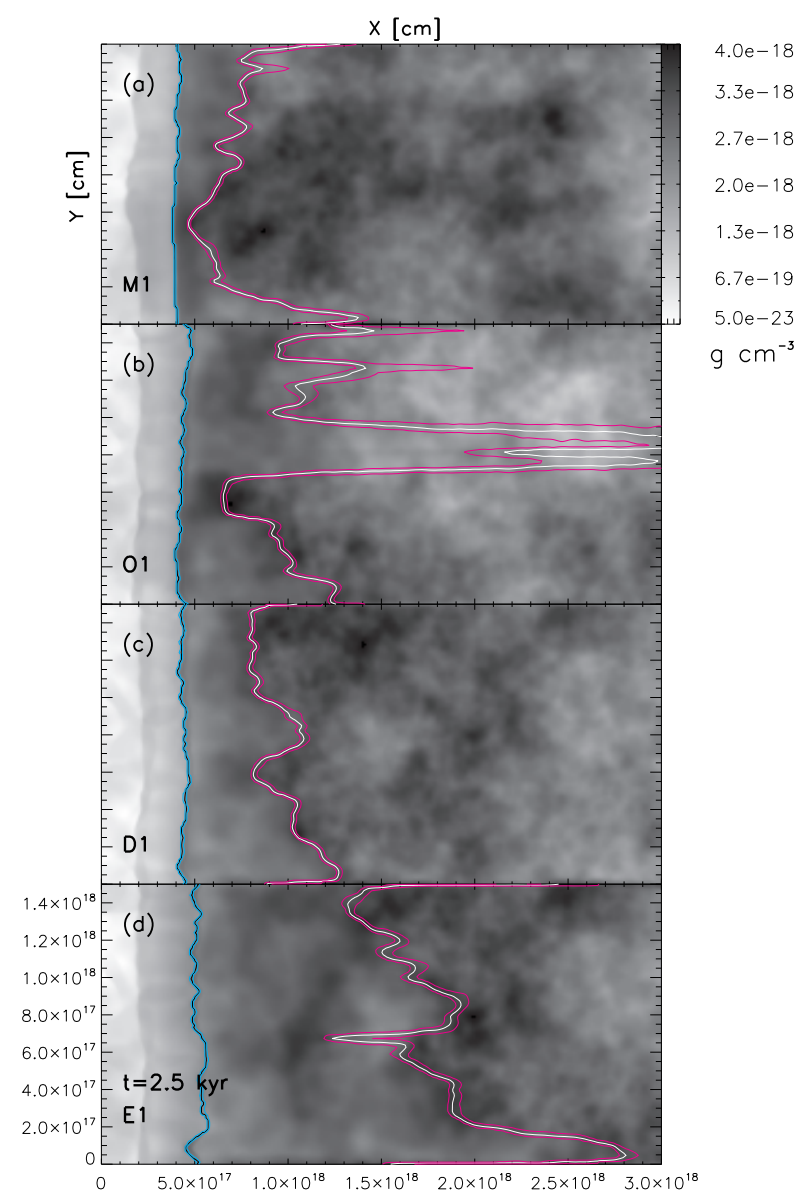

Fig. 1. The $t=2.5 \mathrm{kyr}, x y$-mid-plane density stratifications of models M1, O1, D1 and E1 (with non-zero FUV fields, see Table 1). The density stratifications are shown with the logarithmic gray scale given (in $\mathrm{g} \mathrm{cm}^{-3}$ ) by the top right bar. In the four frames, we show the contour corresponding to an $\mathrm{H}$ ionization fraction of $50 \%$ (black line), which indicates the position of the HI/II ionization front. The blue lines show the width of the HI/II region. The contour corresponding to a $\mathrm{C}$ ionization fraction (white line) of $50 \%$, which indicates the position of the CI/II ionization front, is also shown. The pink lines show the width of the CI/II region. The $x$ and $y$-axes are labeled in $\mathrm{cm}$. The color figure can be viewed online.

and Figure 7 (models M3-E3 and M3B-E3B). These figures show the number of clumps (averaged over $20 \mathrm{kyr}$ time-intervals) as a function of the integration time, obtained with the three chosen cutoff densities.

From Figures 5-7, we see that for the $\rho_{c}=$ $10^{-20} \mathrm{~g} \mathrm{~cm}^{-3}$ cutoff density, the number of clumps first decreases rapidly, and then stabilizes (for $t>$ $20 \mathrm{kyr}$ ) at a value of $\approx 100$ for most of the models. Actually, if for the models with nonzero FUV field we count clumps with neutral $\mathrm{C}$, the number of clumps

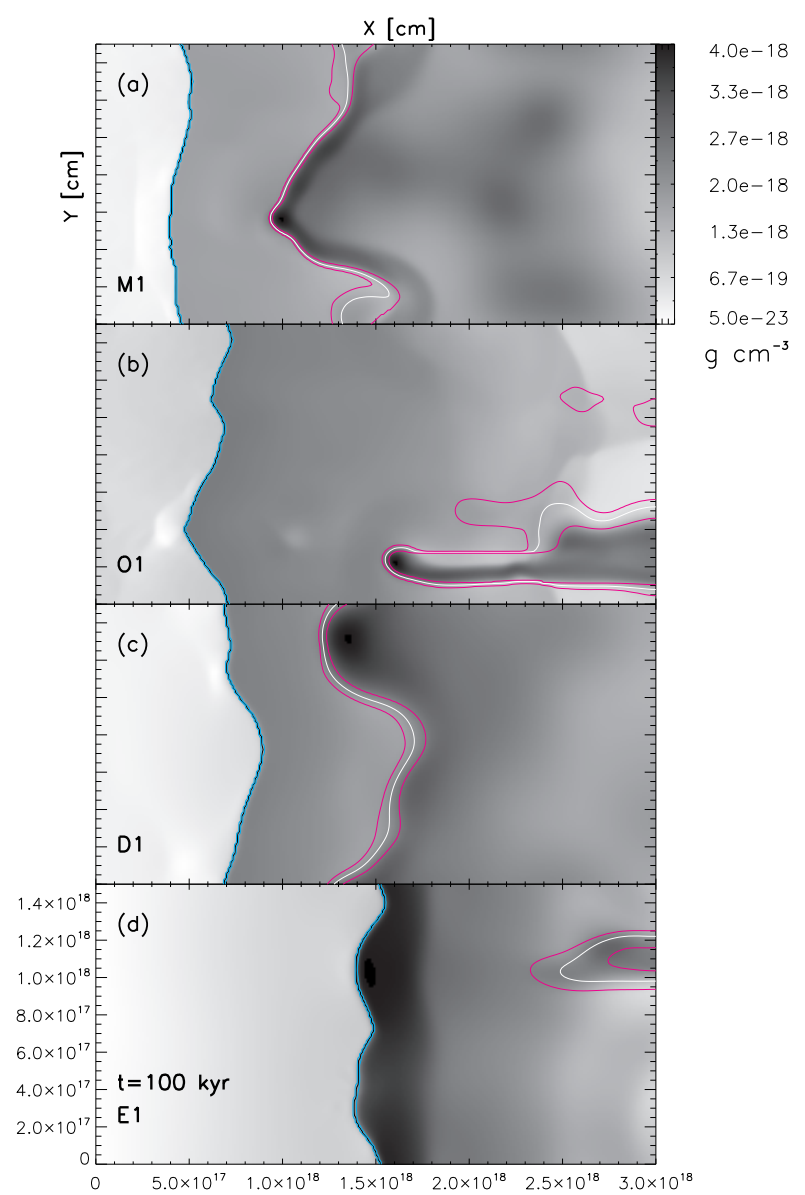

Fig. 2. Same as Figure 1, but for $t=100 \mathrm{kyr}$ (see Table 1). The color figure can be viewed online.

stabilizes at a value of $\sim 200$ (see the top panels of Figures 5-7).

For the $\rho_{c}=10^{-19} \mathrm{~g} \mathrm{~cm}^{-3}$ cutoff density, the number of clumps starts at a value of $\sim 3 \times 10^{3}$, and in all models decreases to $\sim 200$ within the first $\sim 50 \mathrm{kyr}$ of the time-evolution. For larger times, in the models with zero FUV flux (M1B-E1B, M2B-E2B and M3B-E3B, bottom panels of Figures 5, 6, and 7, respectively), the clump number decreases and then stabilizes (for $t>30 \mathrm{kyr}$ ) at a value of $\approx 50$.

For $\rho_{c}=3 \times 10^{-18} \mathrm{~g} \mathrm{~cm}^{-3}$, the models with zero FUV flux take $\sim 50 \rightarrow 70$ kyr to develop the first clump, and by $t=130 \mathrm{kyr}$ they have developed $\sim 40$ clumps. The models with non-zero FUV flux (top panels of Figures 5-7) develop the first clump much earlier, after only $\sim 10 \mathrm{kyr}$, and have about 70 clumps at $t=130 \mathrm{kyr}$.

Even though our models with zero FUV flux (M1B, M2B and M3B) cover a factor of $~ 13$ in EUV photon rates (see Table 1), except for relatively small 


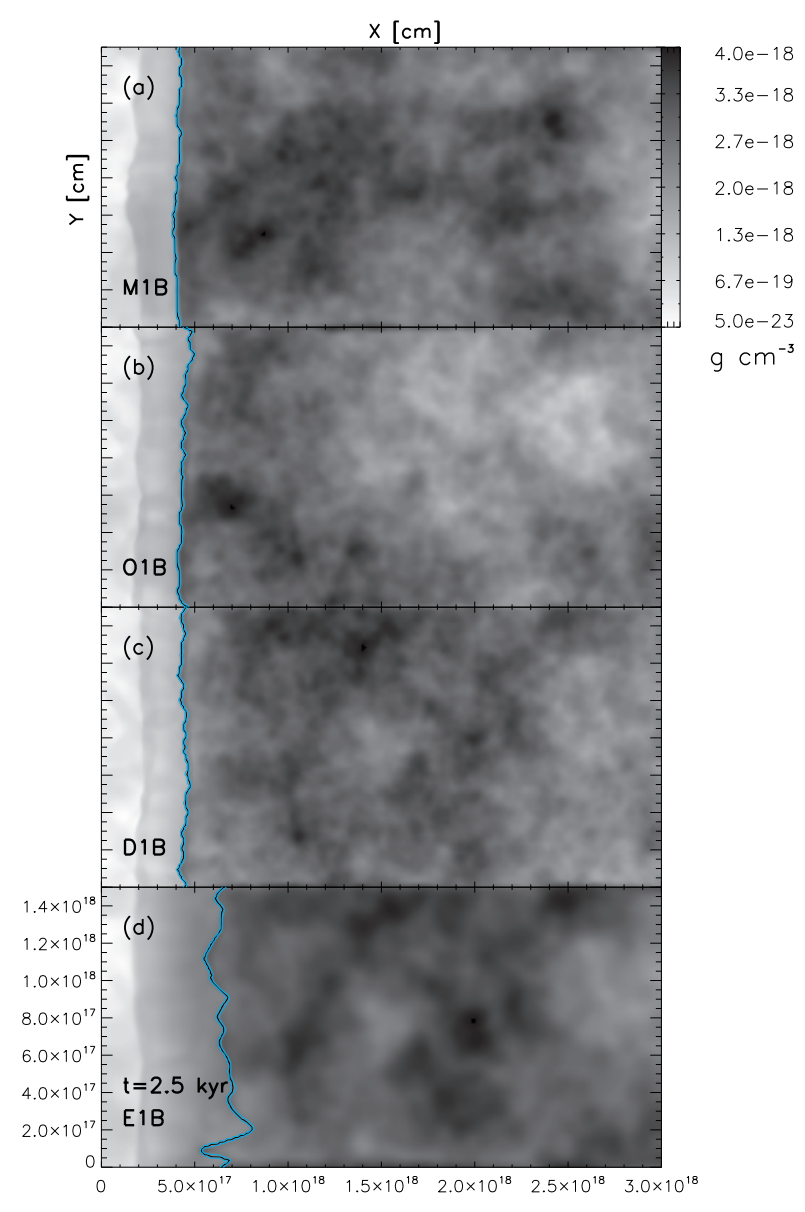

Fig. 3. The $t=2.5 \mathrm{kyr}, x y$-mid-plane density stratifications of models M1B, O1B, D1B and E1B (with zero FUV fields, see Table 1). The density stratifications are shown with the logarithmic gray scale given $\left(\right.$ in $\mathrm{g} \mathrm{cm}^{-3}$ ) by the top right bar. In the four frames we show the contour corresponding to an $\mathrm{H}$ ionization fraction of $50 \%$ (black line), which indicates the position of the HI/II ionization front, and also the contour corresponding to a $\mathrm{C}$ ionization fraction (white line) of $50 \%$, which indicates the position of the CI/II ionization front. The blue lines show the width of the HI/II region. The $x$ and $y$-axes are labeled in $\mathrm{cm}$. The color figure can be viewed online.

effects (e.g., the earlier appearance of high $\rho_{c}$ clumps in model M1B-E1B, see the bottom panel of Figure 5) they show qualitatively similar trends of number of clumps as a function of time. Our models with non-zero FUV flux (M1-E1, M2-E2 and M3-E3, see Table 1) also cover a factor of $\sim 6$ of FUV photon production rates, and these three models also show qualitatively similar time evolution of the number of clumps (top panels of Figures 5-7).

From this, we conclude that for the range of early to late O-type stars chosen, the fragmentation of

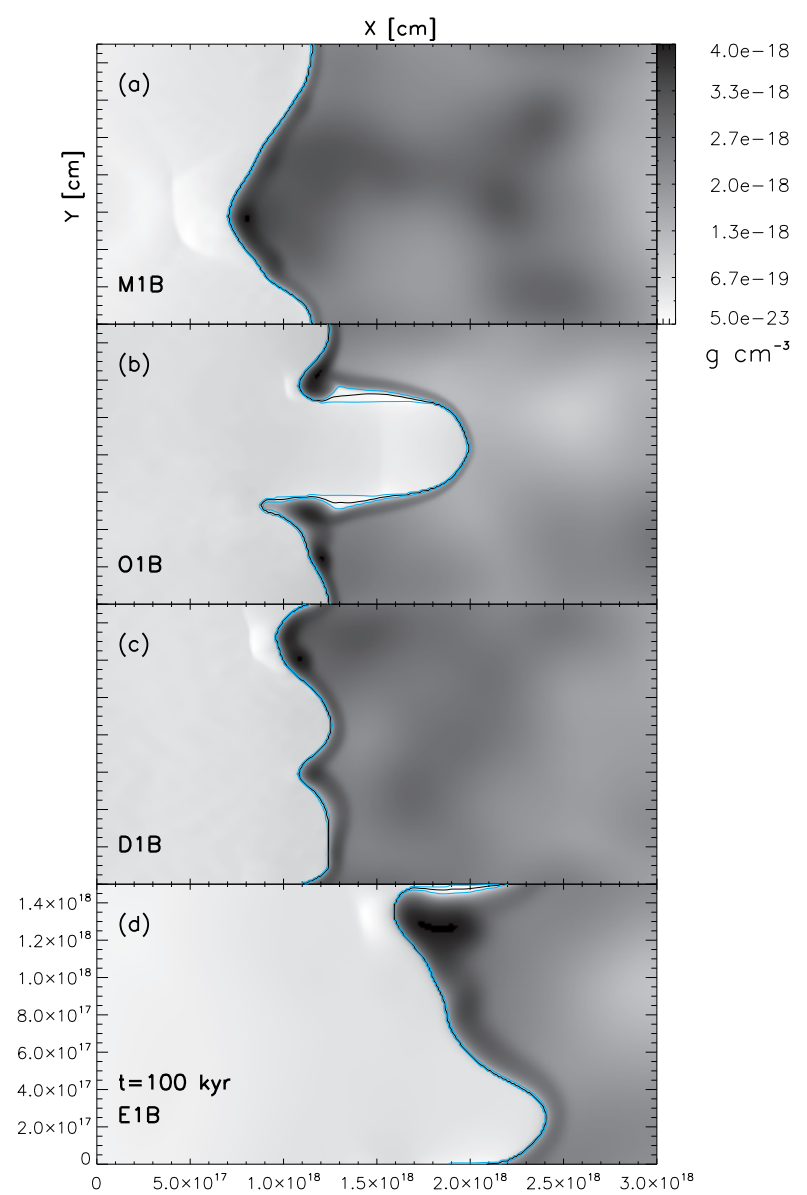

Fig. 4. Same as Figure 3, but for $t=100 \mathrm{kyr}$ (see Table 1). The color figure can be viewed online.

the neutral structure into clumps presents a similar behavior regardless of the spectral subclass of the star. We also see that including the effect of the FUV photons does produce important differences (see the above list of 3 items). The effect of introducing the FUV flux is to increase the number of $\rho_{c}=3 \times 10^{-18} \mathrm{~g} \mathrm{~cm}^{-3}$ clumps (from $\sim 40$ to $\sim 70$ ) at the final, $t=240$ kyr integration time of our simulations.

\subsection{The clump mass distributions}

We now focus on a $t=150 \mathrm{kyr}$ evolutionary time, in which a sizable population of clumps has developed in all models (see Figures 5-7). For the stratifications resulting from all models at this time, we compute the mass distributions of the clumps obtained with the $\rho_{c}=10^{-19}$ and $3 \times 10^{-18} \mathrm{~g} \mathrm{~cm}^{-3}$ cutoff densities. We do not compute the distributions for $\rho_{c}=10^{-20} \mathrm{~g} \mathrm{~cm}^{-3}$ because at $t=150 \mathrm{kyr}$ they only have one clump which includes basically all of the neutral region of the flow. 


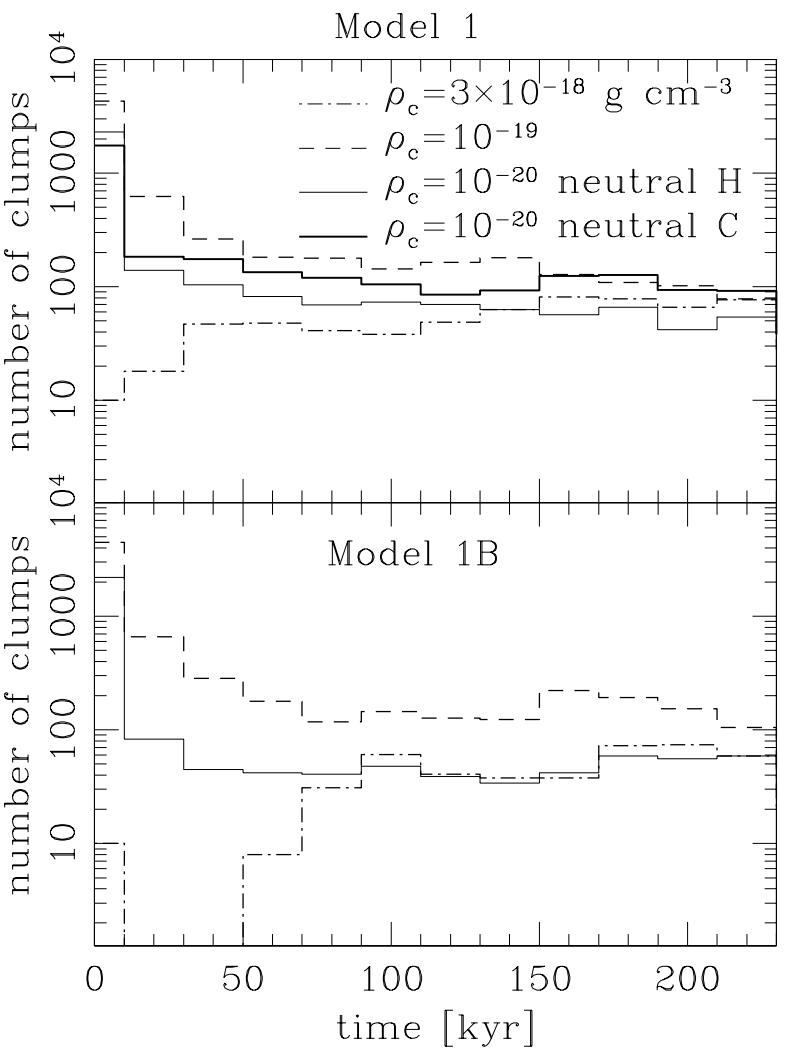

Fig. 5. The top panel shows the number of neutral clumps obtained from models M1, O1, D1 and E1 (with non-zero FUV fluxes, see Table 1) as a function of time, for the three chosen density cutoffs. The bottom panel shows the number of neutral clumps for the same density cutoffs but for models M1B, O1B, D1B and E1B (with zero FUV, see Table 1). In the top panel, the two solid lines correspond to a $\rho_{c}=10^{-20} \mathrm{~g} \mathrm{~cm}^{-3}$ cutoff density, with clumps with neutral $\mathrm{H}$ (thin line) and with neutral $\mathrm{C}$ (thick line).

The resulting clump number vs. mass distributions are shown in Figure 8 (models M1-E1 and M1B-E1B, see Table 1), Figure 9 (models M2-E2 and M2B-E2B) and Figure 10 (models M3-E3 and M3BE3B). If we look at the $\rho_{c}=10^{-19} \mathrm{~g} \mathrm{~cm}^{-3}$ clump mass distributions (top panels of Figures 8, 9, and 10), we observe that the distributions of all models have two clumps in the high mass, $10^{2} \rightarrow 10^{3} M_{\odot}$ bin, and for models with non zero FUV flux 2-4 clumps in the low mass, $10^{-3} \rightarrow 10^{-2} M_{\odot}$ bin.

The distributions of the models with zero FUV flux (M1B-E1B, M2B-E2B and M3B-E3B) have 01 clump in the $10^{-2} \rightarrow 10^{-1} M_{\odot}$ range, while the distributions of the non-zero FUV flux models (M1E1, M2-E2 and M3-E3) have 4-6 clumps in this mass range.

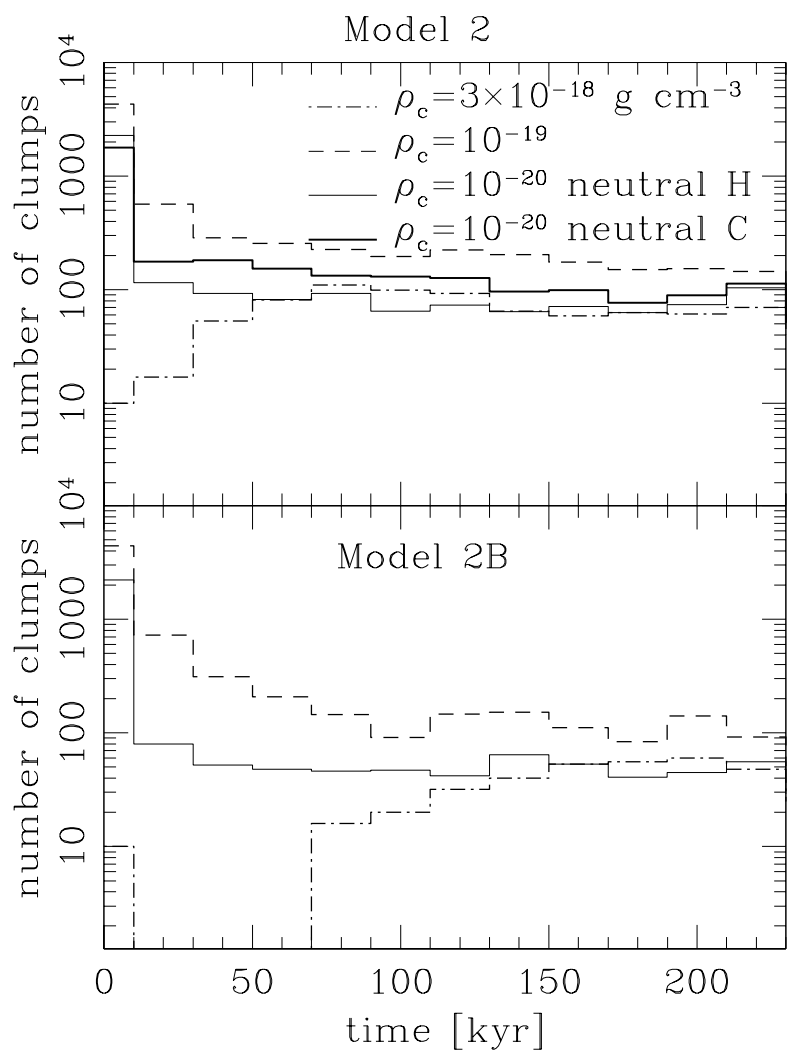

Fig. 6. Same as Figure 5, but for models M2, O2, D2 and E2 (top panel) and M2B, O2B, D2B and E2B (bottom panel).

Therefore, the main effect of including a nonzero FUV field is mostly to enhance the $\rho_{c}=$ $10^{-19} \mathrm{~g} \mathrm{~cm}^{-3}$ number of clumps in the $10^{-3} \rightarrow$ $10^{0} M_{\odot}$ mass range.

If we look at the $\rho_{c}=3 \times 10^{-18} \mathrm{~g} \mathrm{~cm}^{-3}$ clump mass distributions (bottom panels of Figures 8, 9, and 10), we see that the three models with zero FUV flux (M1B-E1B, M2B-E2B and M3B-E3B) show very similar clump mass distributions, with one clump in the $10^{-2} \rightarrow 10^{-1} M_{\odot}$ mass range for model M1BE1B, and no clumps in this mass range for models M2B-E2B and M3B-E3B. In the $10^{-2} \rightarrow 10^{1} M_{\odot}$ range, all of the models with non-zero FUV flux have 5-9 clumps, generally having 2-6 more clumps in this range than the zero FUV models (3 clumps).

Therefore, the presence of an FUV flux allows the formation of more low mass, $\rho_{c}=3 \times 10^{-18} \mathrm{~g} \mathrm{~cm}^{-3}$ clumps, than the zero FUV flux models.

\section{SUMMARY}

In a previous paper (Lora et al. 2009), we have studied the formation of dense clumps in the interac- 


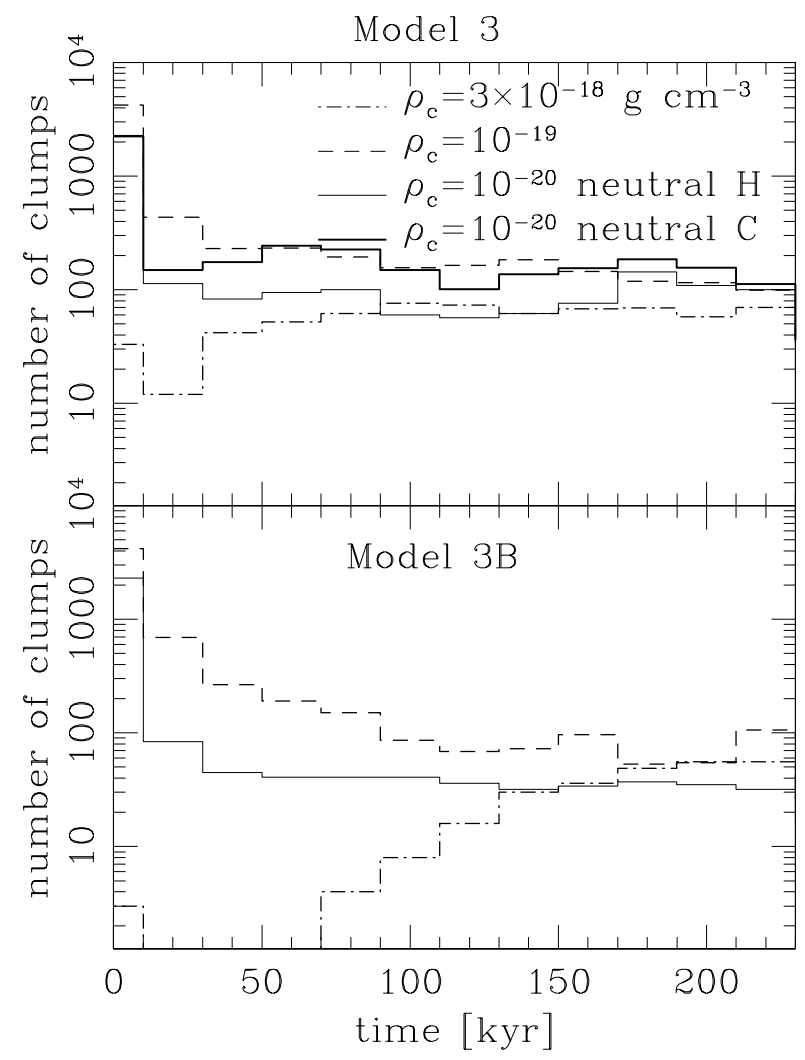

Fig. 7. Same as Figure 5, but for models M3, O3, D3 and E3 (top panel) and M3B, O3B, D3B and E3B (bottom panel).

tion of a photoionizing radiation field with an inhomogeneous medium (with an initial power law spectrum of density fluctuations). The applicability of these models to real astrophysical flows (associated with expanding HII regions) was questionable because of the absence (in the models) of a photodissociation region preceding the HI/II ionization front.

In this work, we present a set of numerical simulations which explore the effect of a FUV radiative field, which produces a photodissociation region outside the HII region. To this effect, we compute 3D simulations which include the photoionization of $\mathrm{H}$ and $\mathrm{C}$, assuming that the $\mathrm{CI} / \mathrm{II}$ ionization front approximately coincides with the outer edge of the photodissociation region (as initially suggested by Richling \& Yorke 2000). Arthur et al. (2011) calculate numerical simulations in which a different approximation is used for determining the outer edge of the photodissociation region, but which lead to similar results.

From our simulations, we obtain the masses and the number of clumps (defined as contiguous regions of density higher than a given cutoff density
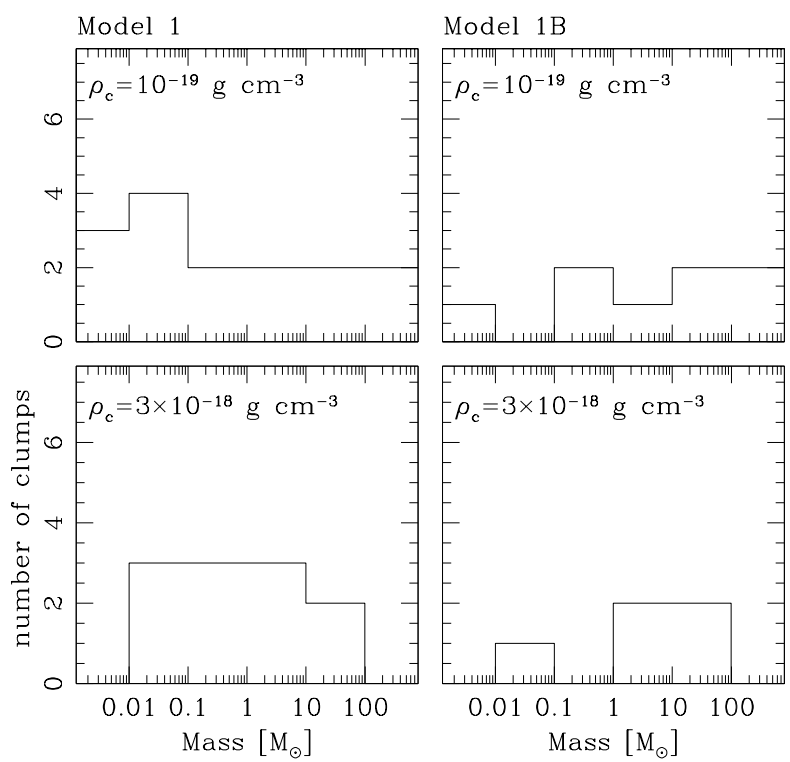

Fig. 8. Neutral clump mass distribution for two cutoff densities $\left(\rho_{c}=10^{-19}\right.$ and $\left.3 \times 10^{-18} \mathrm{~g} \mathrm{~cm}^{-3}\right)$ for the integration time $t=150 \mathrm{kyr}$ for models M1, O1, D1 and E1 (left panels) and M1B, O1B, D1B and E1B (right panels).
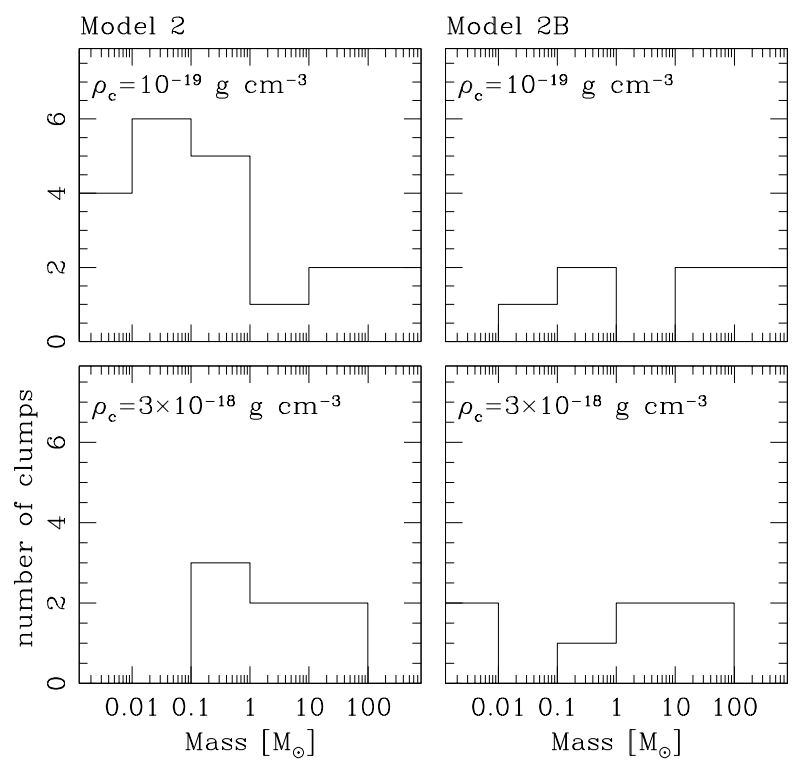

Fig. 9. Same as Figure 8, but for models M2, O2, D2 and E2 (left panels) and M2B, O2B, D2B and E2B (right panels).

$\rho_{c}$, see $\S 3$ ). We calculate models with photoionizing/photodissociating stars at $\approx 1 \mathrm{pc}$ from the computed region, and explore a range from early to latetype O stars (using the EUV and FUV photon rates 

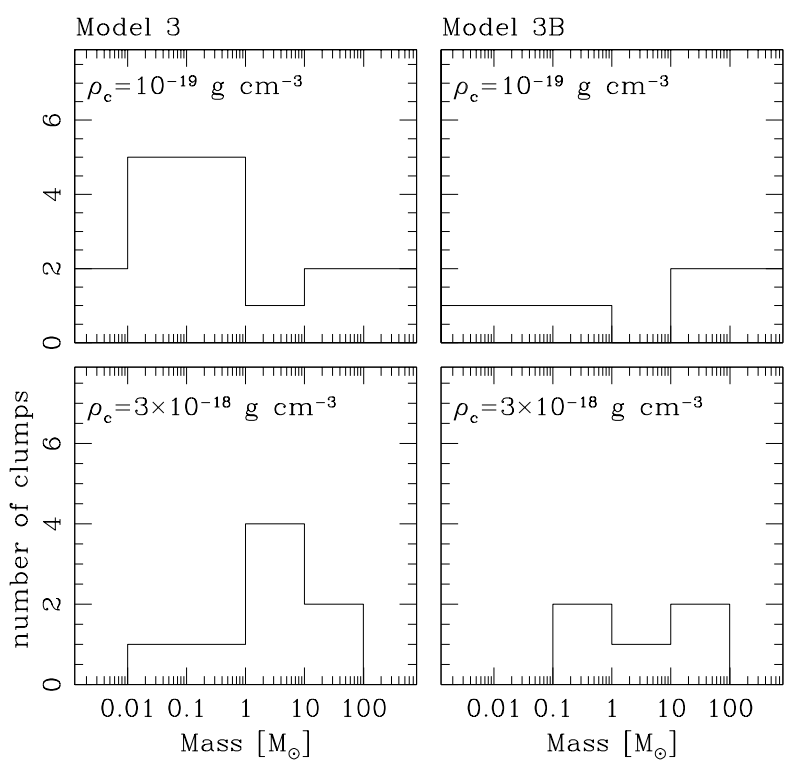

Fig. 10. Same as Figure 8, but for models M3, O3, D3 and E3 (left panels) and M3B, O3B, D3B and E3B (right panels).

presented by Díaz-Miller et al. 1998). We also compute models setting the FUV flux to zero, in order to isolate the effects of having a non-zero FUV flux.

We find that including the photodissociation region produced by a non-zero FUV flux has the following main effects:

- clumps with low cutoff densities $\left(\rho_{c}=\right.$ $10^{-19} \mathrm{~g} \mathrm{~cm}^{-3}$ ) are slightly depleted (see $\S \S 3$ and 4 ),

- denser clumps (with $\rho_{c}=3 \times 10^{-18} \mathrm{~g} \mathrm{~cm}^{-3}$ ) develop earlier than in the models with zero FUV (see Figures 5-7),

- a larger number of dense clumps (with $\rho>\rho_{c}=$ $3 \times 10^{-18} \mathrm{~g} \mathrm{~cm}^{-3}$ ) is produced, and these clumps have a broader mass distribution than in the zero FUV models (see $\S 4$ ).

From this, we conclude that the presence of an outer photodissociation region has an important effect on the formation of dense structures due to the expansion of an HII region in an initially inhomogeneous medium. In particular, including a FUV field leads to the earlier formation of a larger number of dense clumps. This in principle, might lead to the formation of more young stars. However, our simulations do not have the resolution nor do they include the physical processes necessary for determining whether or not the clumps actually collapse to form one or more stars.
VL gratefully acknowledges support from the Alexander von Humboldt Foundation fellowship and H.B-L. We acknowledge support from Conacyt grants 61547, 101356 and 101975. AHC would like to thank the CNPq for partial financial support (307036/2009-0). The authors would also like to thank the anonymous referee for very useful comments.

\section{REFERENCES}

Arthur, S. J., Henney, W. J., Mellema, G., de Colle, F., \& Vázquez-Semadeni, E. 2011, MNRAS, 414, 1747

Bertoldi, F. 1989, ApJ, 346, 735

Bertoldi, F., \& McKee, C. 1990, ApJ, 354, 529

Carlqvist, P. 2010, A\&SS, 327, 267

Carlqvist, P., Gahm, G. F., \& Kristen, H. 2003, A\&A, 403, 399

Dale, J. E., \& Bonnell, I. A. 2011, MNRAS, 414, 321 2012, MNRAS, 422, 1352

Dale, J. E., Bonnell, I. A., \& Whitworth, A. P. 2007, MNRAS, 375, 1291

Dale, J. E., Clark, P. C., \& Bonnell, I. A. 2007, MNRAS, 377,535

Díaz-Miller, R. I., Franco, J., \& Shore, S. N. 1998, ApJ, 501, 192

Ercolano, B., Dale, J. E., Gritschneder, M., \& Westmoquette, M. 2012, 420, 141

Ercolano, B., \& Gritschneder, M. 2011, MNRAS, 413, 401

Esquivel, A., Lazarian, A., Pogosyan, D., \& Cho, J. 2003, MNRAS, 342, 325

Esquivel, A., \& Raga, A. C. 2007, MNRAS, 377, 383

González, R. F., Raga, A. C., \& Steffen, W. 2005, RevMexAA, 41, 443

Gritschneder, M., Burkert, A., Naab, T., \& Walch, S. 2010, ApJ, 723, 971

Gritschneder, M., Naab, T., Walch, S., Burkert, A., \& Heitsch, F. 2009, ApJ, 694, L26

Henney, W. J., Arthur, S. J., de Colle, F., \& Mellema, G. 2009, MNRAS, 398, 157

Hester, J., et al. 1996, AJ, 111, 2349

Lora, V., Raga, A. C., \& Esquivel, A. 2009, A\&A, 503, 477

Lefloch, B., \& Lazareff, B. 1994, A\&A, 289, 559

Lim, A. J., \& Mellema, G. 2003, A\&A, 405, 189

Mackey, J., \& Lim, A. J. 2010, MNRAS, 403, 714 . 2011, MNRAS, 412, 2079

Mac Low, M. M., Toraskar, J., Oishi, J. S., \& Abel, T. 2007, ApJ, 668, 980

Mellema, G., Arthur, S. J., Henney, W. J., Iliev, I. T., \& Shapiro, P. R. 2006, ApJ, 647, 397

Mellema, G., Raga, A. C., Cantó, J., Lundqvist, P., Balick, B., Steffen, W., \& Noriega-Crespo, A. 1998, A\&A, 331, 335

O'Dell, C. R., Henney, W. J., \& Ferland, G. J. 2005, AJ, 130,172 
Oort, J. H., \& Spitzer, L. 1955, ApJ, 121, 6

Raga, A. C., Henney, W., Vasconcelos, J., Cerqueira, A., Esquivel, A., \& Rodríguez-González, A. 2009, MNRAS, 392, 964
Richling, S., \& Yorke, H. W. 2000, ApJ, 539, 258

Vasconcelos, M. J., Cerqueira, A. H., \& Raga, A. C. 2011, A\&A, 527, 86

A. H. Cerqueira and M. J. Vasconcelos: Laboratório de Astrofísica Teórica e Observacional, DCET-UESC, Rodovia Ilhéus-Itabuna, km. 16 - Ilhéus, Bahia, Brazil, CEP 45662-000 (mjvasc, hoth@uesc.br).

A. Esquivel and A. C. Raga: Instituto de Ciencias Nucleares, Universidad Nacional Autónoma de México, Apdo. Postal 70-543, 04510 D. F., Mexico (raga, esquivel@nucleares.unam.mx).

V. Lora: Astronomisches Rechen-Institut Zentrum für Astronomie der Universität Heidelberg, Mönchhofstr. 12-14, 69120 Heidelberg, Germany (vlora@ari.uni-heidelberg.de). 